Revue d'histoire de l'Amérique française

DEVUE D.HISTOIRE DE L'AMÉRIQUE FRANÇAISE

\title{
Maurice Duplessis et sa conception de l'autonomie provinciale au début de sa carrière politique
}

\section{René Durocher}

Volume 23, numéro 1, juin 1969

URI : https://id.erudit.org/iderudit/302851ar

DOI : https://doi.org/10.7202/302851ar

Aller au sommaire du numéro

Éditeur(s)

Institut d'histoire de l'Amérique française

ISSN

0035-2357 (imprimé)

1492-1383 (numérique)

Découvrir la revue

Citer cet article

Durocher, R. (1969). Maurice Duplessis et sa conception de l'autonomie provinciale au début de sa carrière politique. Revue d'histoire de l'Amérique française, 23(1), 13-34. https://doi.org/10.7202/302851ar d'utilisation que vous pouvez consulter en ligne. 


\section{MAURICE DUPLESSIS ET SA CONCEPTION DE L'AUTONOMIE PROVINCIALE AU DÉBUT DE SA CARRIÊRE POLITIQUE ${ }^{1}$}

$\mathrm{Si}$ on interroge les contemporains de Maurice Duplessis ils s'accorderont au moins sur un point, peut-être le seul, à savoir que la défense de l'autonomie provinciale a été le thème principal de sa carrière politique. Mais là s'arrête l'unanimité, comme en témoignent les jugements opposés d'Antonio Barrette et de Georges-Émile Lapalme.

Là où la lutte de Maurice Duplessis fut ardente, constante et éclairée à la fois, là où il a rendu les plus grands services aux Canadiens et aux citoyens de Québec, c'est par sa défense vigoureuse et soutenue de l'autonomie des provinces (...)

Maurice Duplessis, après son avènement comme Premier ministre de la province en 1936, renoua la lignée des défenseurs de l'autonomie avec fermeté et constance. ${ }^{2}$

Georges-Émile Lapalme, l'ancien chef de l'opposition - qui l'est resté même lorsque son parti était au pouvoir - ne peut que différer d'avis avec A. Barrette.

Admettons en toute objectivité, d'abord et avant tout, que Maurice Duplessis a réellement inventé l'autonomie provinciale, même si avant lui on l'avait invoquée. (...)

Autonomie électorale, autonomie négative, autonomie verbale, autonomie saugrenue, autonomie de remplissage, autonomie du néant. Oui, je sais. Mais y a-t-il eu quelqu'un qui l'ait mieux dorée que lui ?

(...) Il nous a tous traumatisés pour le mieux, et de cet homme qui ne lisait rien et n'était pas un penseur est sorti un mot dont nous avons fini par faire une réalité politique et matérielle. Ses disciples survivants diront qu'il a lancé plus qu'un mot, mais une idée. Je ne chicanerai pas sur les mots eux-mêmes car les siens nous ont fait assez souffrir (...) il [Duplessis] a été un point de ralliement de tout un peuple autour d'un mot qu'il avait rendu prestigieux et dangereux. ${ }^{3}$

${ }^{1}$ Conférence prononcée à la réunion générale de l'Institut d'histoire de l'Amérique française, à Québec, le 17 mai 1969.

2 Antonio Barrette, Mémoires (Montréal, 1966), 384, 386.

3 Georges-Emile Lapalme, "Duplessis vu par Lapalme. Extraits des mémoires inédits de l'ancien chef libéral," Magazine Maclean 9, 2 (février 1969) : 16 . 
En nous servant d'ouvrages comme ceux de Rumilly, Quinn et Roberts, des journaux de l'époque, des documents publics et de quelques lettres personnelles de Duplessis au premier ministre d'Ontario, Mitchell Hepburn, que nous avons retrouvées aux Archives provinciales d'Ontario, nous tenterons de rappeler les principales étapes de sa carrière, les grands thèmes de sa pensée politique et enfin, de faire la genèse de ce mot, ou de cette idée: l'autonomie provinciale dans la pensée et l'action de Duplessis de 1927 à 1939.

Quand on s'appelle Maurice Duplessis, qu'on voit le jour à Trois-Rivières à la fin du dix-neuvième siècle et qu'on est le fils d'un homme qui a été député conservateur de 1886 à 1900 à l'Assemblée législative du Québec, on risque fort de naître conservateur et de le demeurer toute sa vie durant. Nous ne voyons guère d'autres raisons pour expliquer qu'un jeune avocat, intelligent et ambitieux comme Maurice Duplessis, fasse campagne pour un candidat conservateur en 1921, soit sous l'égide de Meighen, au lendemain de la conscription ! Après une tentative infructueuse en 1923, Duplessis réussit à se faire élire député conservateur à Trois-Rivières en 1927.

Duplessis pour être élu devait compter bien plus sur sa réputation personnelle et sur la défense des intérêts locaux que sur le prestige et le programme du parti conservateur discrédité malgré tous les efforts d'Arthur Sauvé pour se dissocier des conservateurs fédéraux. Dès son élection comme candidat conservateur il esquisse les grandes lignes de son programme:

Les Trois-Rivières d'abord, les Trois-Rivièrres ensuite, les Trois-Rivières toujours. Il a porté, écrit le correspondant du Devoir, certaines accusations contre le régime actuel, entre autres celle de protéger les gros au détriment des petits, et de n'avoir de faveurs que pour les millionnaires. Il promet de toujours mettre l'intérêt public avant les questions de parti et de défendre l'intérêt et le capital des Trois-Rivières par-dessus tout et malgré tout. ${ }^{4}$

Il ne manque qu'un thème, celui de l'observance du dimanche qui suscite beaucoup d'inquiétude dans les milieux bien-pensants de la Mauricie. Duplessis saura exploiter à fond ce sujet qui met

${ }^{4}$ Le Devoir, 27 avril 1927. 
en cause les valeurs les plus chères de la population et il nous semble que Rumilly n'a pas tort d'y voir un élément déterminant de sa première victoire. Autant il est difficile pour un conservateur de se faire élire au Québec autant il est facile pour un homme de grand talent comme Duplessis de monter dans ce parti peu encombré. Il faut suivre Duplessis au début de sa carrière pour comprendre comment il a su rallier autour de lui non seulement les conservateurs mais les nationalistes et les libéraux dissidents.

Dès ses premières interventions en Chambre il s'attire les éloges des observateurs politiques, en particulier ceux du Devoir qui ne lui ménageront pas les encouragements et la publicité pendant la période que nous étudions. Duplessis est un remarquable opposant, qui a toujours l'art de poser des questions opportunes, de formuler des critiques incisives qui ternissent l'image du gouvernement Taschereau. En plus de mettre à profit ses connaissances juridiques pour éplucher les textes de loi et se sortir du maquis de la procédure il fait preuve d'un flair politique certain et d'une prudence de langage indispensable à celui qui veut poursuivre une longue carrière et rallier autour de lui une majorité. Il s'engage,... sans se compromettre. Ainsi il s'attaque avec véhémence aux grandes compagnies, il dénonce les "trusts", le "capitalisme ventriloque" tout en prenant soin de dire qu'il favorise le capital sain respectueux de nos lois; ou encore, il se contente de formuler des critiques sans proposer d'alternatives autres que des améliorations de détail ou des vœux... pieux. Peu importe, l'habileté et l'efficacité de son travail parlementaire sont tels qu'à peine élu on suggère son nom comme futur chef de l'opposition.

La première préoccupation d'un politicien digne de ce nom c'est sans conteste de conserver son siège de député. Chaque fois qu'il est question de Trois-Rivières, Duplessis intervient pour défendre les intérêts de ses électeurs, soit contre le gouvernement provincial qui accapare les revenus municipaux, soit contre les grosses compagnies qui jouissent, grâce à Taschereau, d'exemptions de taxes scandaleuses. Sans cet inlassable dévouement, il n'aurait peut-être pas obtenu les quarante et une voix de majorité 
qui lui permirent de revenir siéger à l'Assemblée au lendemain de l'élection de 1931.

L'action parlementaire de Duplessis se situe cependant dans un cadre plus large que la simple défense des intérêts de sa région. Tout au long des sessions il attaque avec beaucoup de succès le gouvernement Taschereau "autocratique" et défenseur des "gros intérêts".

$\mathrm{Au}$ lendemain de sa première session, il affirme avoir constaté:

... que la législature de Québec, au lieu de se faire l'écho

des aspirations populaires, se fait l'écho des gros intérêts

personnels au détriment de ceux du peuple (...) Le gouvernement a deux "machines", une qui fait du cent à l'heure et une brouette. La première sert à conduire les bills des grosses compagnies, la seconde transporte les bills populaires. ${ }^{5}$

D'une manière générale, le député de Trois-Rivières se donne le rôle de défenseur des libertés du peuple ou mieux de protecteur des "petits" (municipalités, contribuables, petits propriétaires, petits commerçants, ouvriers et agriculteurs). L'inquiétude et la misère provoquées par la crise économique donnent beaucoup de force aux attaques incessantes de Duplessis contre ce qu'il appelle la dictature économique et l'autocratie gouvernementale.

En 1933, Duplessis s'était bâti une réputation telle qu'il pouvait aspirer à la direction du parti conservateur provincial. Certains membres du parti, tels A. Guertin, L. Barré, R.L. Calder et surtout Camillien Houde qui ne prisaient guère son ambition et son conservatisme au point de vue économique et social, lui suscitèrent un adversaire en la personne d'Onésime Gagnon.

Gagnon étant député conservateur à Ottawa, rien de plus facile ni de plus habile que de faire courir la rumeur qu'avec lui le parti provincial serait soumis à R.B. Bennett et qu'en définitive cela servirait très mal les intérêts des conservateurs provinciaux. En réalité, un seul ministre fédéral appuyait Gagnon, et son principal allié C. Houde n'était pas précisément dévoué aux intérêts des fédéraux! Il convient aussi de souligner que Duplessis à cette époque entretenait de bons rapports avec

5 Ibid., 10 septembre 1928. 
les fédéraux. Ainsi, en 1928, il assiste au banquet Bennett à Montréal, en '29 il participe à un congrès des conservateurs fédéraux à Québec, en '31 il aide le conservateur Charles Bourgeois à remporter le siège fédéral de Trois-Rivières lors d'une élection complémentaire. À la convention même, il s'empresse de présenter la première résolution qui se lit comme suit:

Cette convention présente ses hommages au premier ministre du Canada, l'hon. R. B. Bennett: elle le remercie du travail qu'il s'impose dans le règlement des difficultés qui troublent le pays et l'assure de son entière volonté de collaboration et de sa coopération. ${ }^{6}$

Si Duplessis a triomphé facilement en 1933 ce n'est pas à cause de son opposition aux conservateurs fédéraux ni à cause de sa contribution insignifiante au programme du parti mais bien, comme le fait remarquer un rédacteur de l'Action Nationale, parce qu'il est passé maître dans le travail d'organisation, parce qu'il "connaît le hommes et [que] son flair politique lui indique de façon certaine dans quel sens il doit orienter ses efforts. Prudent et maître de lui-même, il ne laisse rien au hasard" ". Le même observateur ne manque pas de perspicacité lorsqu'il écrit du nouveau chef de l'opposition:

Jusqu'ici, c'est surtout par les qualités secondaires de l'homme d'Etat que Duplessis s'est assuré les succès qu'il a connus: talent d'organisation, facilité d'élocution, souplesse d'esprit, subtilité de jugement, sens juridique (...) Il lui a manqué une culture générale suffisamment approfondie, une ampleur de vision capable d'envisager les problèmes sous tous leurs aspects, et, peut-être, le feu sacré des grands convaincus. C'est ce qui explique pourquoi il n'a pu créer encore de programme strictement original, ni attacher son nom et son talent à aucune grande question nationale.

Et l'auteur de conclure, malgré tout: "Son passé porte assez de promesses pour autoriser bien des espoirs." 8

L'analyse rigoureuse de sa pensée économique et sociale, avant comme après 1933, n'autorisait guère, à notre avis, ces espoirs qui seront si cruellement déçus. Les libéraux réformistes et les nationalistes, exaspérés par le régime Taschereau, se

6 Ibid., 4 octobre 1933.

7 Anonyme, "Maurice Duplessis", L'Action Nationale, 2 (novembre 1933) : 173.

8 Ibid., 173-174. 
rallieront à Duplessis et lui serviront de tremplin en 1935-1936. Ils seront victimes autant de son habileté que de leur bonne foi un peu naïve.

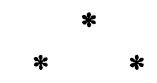

Le credo politique de Maurice Duplessis, comme sa pensée économique et sociale, est essentiellement conservateur et comprend tous les articles de notre tradition. Lors du deuxième congrès de la langue française, à Québec en 1937, il aura l'occasion de synthétiser sa pensée sur le sujet:

Après la religion, la langue est notre plus fort lien d'attache. (...) Oui la race canadienne-française est loyale à la Couronne et au bilinguisme décrété par le pacte de la Confédération.

(...)

... si quelques-uns avaient le malheur de prêcher l'isolement, je suis certain qu'ils seraient réprouvés par leurs concitoyens. ${ }^{9}$

L'autonomie provinciale, implicite dans l'idée de pacte confédératif, constitue sur le plan politique une valeur traditionnelle du Canada français. Depuis Georges-Etienne Cartier et Antoine-Aimé Dorion, en principe, à peu près tous les Canadiens français reconnaissent l'importance de cette question. Duplessis qui, tout en acceptant la nécessité de certains changements, disait de lui-même: "Je suis un traditionnaliste, dans ce sens que la tradition doit être un fondement sur lequel nous devons édifier notre œuvre", ${ }^{10}$ ne pouvait qu'assumer cette valeur: l'autonomie provinciale.

Même si comme simple député ou comme chef de l'opposition, Maurice Duplessis semble plus préoccupé par l'autonomie municipale constamment rognée par le régime Taschereau, que par l'autonomie provinciale, son attachement à cette dernière cause ne fait aucun doute, du moins au niveau des principes. Dans ses discours, il emploie des expressions comme: "l'autonomie qui est pour ainsi dire l'âme de la race". ${ }^{11}$ Souvent pour expliciter sa pensée, il dénonce l'union législative: "Nous avons et nous devons garder une Confédération de provinces autonomes, car

\footnotetext{
${ }^{9}$ Maurice Duplessis, in Deuxième Congrès de la langue française au Canada (Québec, 1938), 172-173 et 347 .

${ }^{10} \mathrm{Le}$ Devoir, 5 décembre 1982.

11 Ibid., 2 juillet 1935.
} 
le système opposé, l'union législative, serait excessivement dangereuse et des plus dommageables !" 12 Il invoque, comme il convient, le "pacte confédératif" et trahit son profond conservatisme lorsqu'il s'oppose à toute modification de l'A.A.N.B., car dit-il: "Bien que la Constitution ne soit pas parfaite, elle nous procure beaucoup plus d'avantages que d'inconvénients. En voulant l'améliorer, on pourrait ouvrir la porte à de plus grands dangers". ${ }^{13}$

Duplessis, foncièrement pragmatique, n'aime guère les théories et c'est à travers son attitude face à des problèmes concrets, tels la stratégie à adopter vis-à-vis les partis fédéraux ou devant certaines mesures législatives qu'on a chance de découvrir sa pensée autonomiste.

Depuis 1867, l'affiliation à un parti politique influence plus ou moins, selon les circonstances et les hommes en présence, les relations fédérales-provinciales. On peut dire que depuis l'arrivée des conservateurs à Ottawa, Duplessis prêche avec constance la bonne entente et la coopération entre les gouvernements d'Ottawa et de Québec. Il faudrait collaborer avec le gouvernement central pour régler notamment les problèmes relatifs à la surcapitalisation, aux pensions de vieillesse, au transport, à l'enseignement technique et aux pêcheries, car explique Duplessis :

Il est permis de combattre le gouvernement fédéral pendant les élections mais il n'est pas permis à celui qui veut avant tout le bien de sa province et de son pays d'oublier que le gouvernement - qu'il soit libéral ou qu'il soit conservateur - représente l'autorité avec laquelle il est nécessaire de transiger la meilleure solution possible aux nombreux problèmes qui sollicitent notre attention (...) Les problèmes qui nécessitent la collaboration du Fédéral et du provincial auront plus de chance d'être favorablement résolus si le ler ministre de la province apporte à leur discussion un esprit de conciliation, de courtoisie et de bonne entente ...14

Taschereau pour qui le "rouge à Québec, rouge à Ottawa" a presque valeur de dogme a quelquefois tendance à se faire du capital politique aux dépens des conservateurs fédéraux. Duplessis, de son côté, essaie discrètement de défendre son parti

12 Ibid., 8 novembre 1935.

13 Ibid., 28 février 1934.

14 Ibid., 13 janvier 1933. 
contre ces attaques partisanes. Mais avec l'impopularité croissante de Bennett et l'émergence d'une coalition des conservateurs provinciaux et des membres de l'Action Libérale Nationale, Duplessis modifie un peu sa stratégie en mettant de plus en plus l'accent sur l'idée que l'autonomie provinciale signifie, en pratique, une nette distinction entre les partis fédéraux et provinciaux. Duplessis qui se méfie de l'intervention des libéraux fédéraux susceptibles d'aider efficacement leurs alliés du Québec déclare: "M. Taschereau, qui parle à tort et à travers de l'autonomie provinciale, prend tous les moyens pour faire disparaître notre autonomie en pratiquant la fusion électorale."15 Quant à lui, il promettra peu avant son accession au pouvoir:

Le gouvernement que je dirigerai ne s'occupera pas de politique fédérale ni pour un parti ni pour l'autre parce que mêlant les deux politiques, on met de côté l'esprit du pacte confédératif pour préconiser l'Union législative qui serait le plus funeste désastre pour notre province. L'Union législative serait la mort de notre province. ${ }^{16}$

Sur le plan des principes Duplessis fait preuve d'un autonomisme très orthodoxe et les considérations partisanes ne l'influencent pas de manière excessive. Cependant, si on analyse ses interventions à l'Assemblée ou ses discours publics tels que rapportés par le Devoir de 1927 à 1936, on trouvera quelques prises de position assez étonnantes pour qui proclame que l'autonomie est "l'âme de la race" ou qu'elle lui est aussi précieuse que la "prunelle de ses yeux".17

En 1929, Duplessis s'oppose à l'institution d'une taxe de vente à Montréal alléguant, entre autres raisons, que c'est une taxe indirecte, donc réservée au gouvernement fédéral. ${ }^{18}$ Heureusement que Taschereau ne poussait pas le respect de la Constitution jusque-là!

Son attitude n'est guère plus autonomiste en matière de droits sur la radio. En 1929, le premier ministre Taschereau avait pris l'initiative de présenter une loi affirmant très explicitement et très concrètement les droits du Québec dans ce

\footnotetext{
15 Cité par Robert Rumilly, Histoire de la Province de Québec (Montréal, 1963), 34: 126.

${ }_{16} \mathrm{Le}$ Devoir, 20 juillet 1936.

17 Ibid., 13 janvier 1936.

18 Ibid., 7 mars 1929.
} 
domaine litigieux et important. ${ }^{19}$ Duplessis critiqua ce projet car il estimait qu'il niait les droits du fédéral. ${ }^{20}$ Taschereau, freiné dans son action par le fédéral, avertit le ministre de la Marine, Duranleau, qu'il était prêt à soumettre, si nécessaire, cette cause aux tribunaux pour défendre les droits du Québec. Duplessis souhaite de son côté qu'on négocie avec le fédéral plutôt que de le provoquer. ${ }^{21}$ Après le jugement du Conseil privé qui a tranché le litige entièrement en faveur d'Ottawa, Taschereau s'obstine à ne pas abroger la loi de 1929. Maurice Duplessis s'en scandalise, car dit-il: “Cette loi est un défi à la Cour suprême et au Conseil privé. Nous avons trop de lois inopérantes qui entraînent le mépris des tribunaux et des lois. C'est une invite au bolchevisme." 22

La défense de l'autonomie provinciale devient extrêmement difficile lorsqu'il en coûte des millions de dollars aux citoyens québécois pour affirmer leurs droits. On le constata lorsqu'en 1927 le gouvernement fédéral vota la loi des pensions de vieillesse dont il s'engageait à défrayer $50 \%$ du coût. Taschereau, estimant que ce domaine relevait de la compétence provinciale, refusa de participer à ce programme à frais partagés. Lorsque la part du fédéral fut portée à $75 \%$ en 1931 , les provinces récalcitrantes cédèrent peu à peu. Seul le Québec résistait et continuait à défrayer l'assistance aux vieillards de la province en plus de payer des impôts à Ottawa pour le "programme national". La tentation était forte pour le chef de l'opposition de s'apitoyer sur le sort "de nos vieux et de nos vieilles" et de blâmer le gouvernement Taschereau. Duplessis, après avoir demandé de négocier avec Ottawa, finit par prôner qu'on accepte purement et simplement la loi fédérale. Même si le principe de la loi n'est pas parfait, dit Duplessis, "la province paie le tiers des pensions de vieillesse et devrait bénéficier de la part qui lui revient." ${ }^{23}$ La pression exercée avec ce genre d'argument fut telle que Taschereau dut se résigner en 1936 à accepter la loi fédérale.

19 Québec, Statuts de Québec, 19 Geo. V. (1929) chapitre 31: 117-118.

20 Le Devoir, 20 mars 1929.

21 Ibid., 23 janvier 1931.

22 Ibid., 31 janvier 1935.

23 Le Devoir, 12 janvier 1934. 
Entre le vote de la loi et son application, il y eut des élections qui portèrent l'Union Nationale au pouvoir. Duplessis se fit une gloire d'avoir été le distributeur des premiers chèques de pension de vieillesse sans s'apercevoir que, du même coup, il avait avalisé une atteinte grave à l'autonomie provinciale. ${ }^{24}$

L'autonomie provinciale fait partie de nos valeurs traditionnelles et c'est à ce titre que, Duplessis essentiellement conservateur, l'assume. L'autonomie repose sur le postulat qu'en 1867 il y eut un pacte confédératif. Jamais Duplessis n'a développé sa pensée à propos de ce pseudo-pacte. Jusqu'en 1936 l'autonomie, malgré les professions de foi verbales de Duplessis, n'est pas en pratique une affaire importante pour lui. Lorsque la théorie du "pacte" est confrontée à des mesures concrètes, Duplessis se contente de suivre Taschereau plus vigilant et plus lucide que lui, ou il ne se prononce pas, ou il adopte des positions qui ne sont guère autonomistes: taxe de vente, radio, pensions de vieillesse.

Bref, Duplessis jusqu'en 1936, n'a absolument rien apporté à la cause de l'autonomie provinciale, sauf une chose: la création d'un parti exclusivement québécois, l'Union Nationale. En 1935, Duplessis a dû se résigner, semble-t-il, ${ }^{25}$ à une alliance avec l'Action Libérale Nationale car c'était le seul moyen de renverser le régime libéral au pouvoir depuis 1897. Avec l'arrivée des libéraux dissidents et de nationalistes, Duplessis, même s'il restait imbu de conservatisme, devait nécessairement se dissocier du parti conservateur. Grâce à Gouin et à ses disciples on renoua avec l'idée - fort équivoque d'ailleurs - d'un parti national qui avait avorté en 1872 et qui n'avait eu qu'une précaire et éphémère existence au temps de Mercier.

Même si en 1936 Duplessis a éliminé Paul Gouin, il garde le principe d'un parti exclusivement provincial. La campagne qui lui permettra d'accéder au pouvoir porte sur les scandales du régime Taschereau et sur la crise économique. Il n'est pratiquement pas question d'autonomie provinciale, car l'opposition

24 Cf. Rapport de la Commission royale d'enquête sur les problèmes constitutionnels [Rapport Tremblay] (Québec, 1956), I: 101-105.

${ }^{25}$ Pierre Van Der Donckt, L'Action Libérale Nationale. Thèse de M.A. (Histoire), (U. de M., 1966). 
n'avait guère de griefs à formuler contre la politique du régime libéral à ce sujet. ${ }^{26}$ Certes, à la conférence fédérale-provinciale de décembre 1935, Taschereau, abattu par sa quasi défaite du mois précédent, avait fait preuve d'une certaine faiblesse vis-à-vis ses amis fédéraux qui l'avaient aidé à résister au raz de marée de l'union Gouin-Duplessis. ${ }^{27}$ Mais comme cela n'avait entraîné aucune conséquence fâcheuse, on oublia vite cet incident.

Duplessis, qui essayait de réunir autour de lui, conservateurs, libéraux et indépendants sous l'égide de l'Union Nationale, s'en tint exclusivement à des questions provinciales. Il affirma avec insistance la nécessaire séparation à établir entre les partis fédéraux et provinciaux. Il promit de collaborer avec le gouvernement fédéral, qu'il soit bleu ou rouge. Il répéta à maintes reprises: "Je ne suis pas bleu, je ne suis pas rouge, je ne suis pas tory, je suis de tout mon cour et de toute mon âme, national." 28

La création d'un parti exclusivement québécois constitue un événement majeur dans l'histoire du Québec. L'Union Nationale, à cause de son idéologie conservatrice et parce que son seul champ d'action était la province de Québec, ne pouvait qu'être fortement autonomiste.

Si on accepte l'idée que l'autonomie du Québec est essentielle à l'épanouissement de la collectivité canadienne-française et que cette autonomie est mieux protégée par un parti exclusivement québécois que par un parti provincial inféodé ou même associé à un parti fédéral, on reconnaîtra que Duplessis, malgré lui jusqu'à un certain point et par la force des circonstances, a rendu un très grand service au Québec en faisant de l'Union Nationale un instrument politique efficace et durable.

Duplessis n'a rien d'un théoricien et c'est dans l'exercice du pouvoir qu'il sera amené à donner un contenu positif à sa

26 J.-B. Thivierge, éd., Le Catéchisme des électeurs (Montréal, 1936). Il s'agit du programme de l'Union Nationale.

27 Canada, éd., Conférence du Dominion et des provinces. Compterendu des délibérations, Ottawa 9-13 décembre 1935 (Ottawa, 1936), 11, $35-36$.

${ }^{28}$ Le Devoir, 3 août 1936. 
pensée autonomiste et qu'il en viendra peu à peu à en faire grâce aux circonstances: crise, guerre - son principal cheval de bataille.

Il lui faudra un certain temps avant de s'intéresser à cette question, car après son accession au pouvoir, Duplessis est débordé par des tâches urgentes et difficiles: constitution de son cabinet, état précaire des finances de la province et "l'opération nettoyage".

Il a promis de collaborer avec le fédéral. Effectivement, un mois après son triomphe, le nouveau gouvernement québécois signe avec Ottawa une entente par laquelle les deux gouvernements s'engagent à dépenser chacun $\$ 5,250,000$ pour réduire le chômage dans la province. L'accord est d'autant plus avantageux que les municipalités n'auront aucune contribution à fournir et que le choix des travaux ressortit au gouvernement provincial. ${ }^{29}$

Duplessis, dans l'opposition, avait suggéré à Taschereau, non seulement de collaborer avec le fédéral mais aussi avec les autres provinces notamment pour régler la crise de l'industrie papetière. Le conseil était superflu car depuis très longtemps Taschereau, à l'instar de ses prédécesseurs notamment Gouin et Mercier, était conscient de cette nécessité. Même si dans les années 1935-1936 les relations du Québec et de l'Ontario, ou plus exactement les relations entre les deux premiers ministres: Taschereau et Hepburn, s'étaient détériorées très sérieusement au point de paralyser la coopération des deux provinces centrales, cela ne pouvait qu'être provisoire car trop de forces (géographiques, économiques voire même politiques) commandaient, surtout en cette période de crise économique, une étroite collaboration entre les deux provinces centrales.

Dès l'arrivée au pouvoir de Duplessis, le premier ministre d'Ontario, Mitchell Hepburn, lui écrit pour le féliciter de sa victoire et lui offrir sa collaboration. ${ }^{30}$ Duplessis qui avait très bien accueilli l'élection de Hepburn en 1934, même s'il était un 20.

29 R. Rumilly, Histoire de la province de Québec (Montréal, 1966), 36 :

30 M. F. Hepburn à M. Duplessis, 26 août 1936. Hepburn Papers, Private, 1936. Provincial Archives of Ontario. (P.A.O.). 
libéral, accepte volontiers l'offre de son homologue ontarien et promet de le rencontrer dès que possible. ${ }^{31}$

D'octobre 1936 à avril 1937 Duplessis et Hepburn se rencontrent deux fois et on peut percevoir les effets de leur collaboration dans les domaines de l'exploitation des ressources naturelles et de la législation du travail. A trois reprises, ils adoptent des positions identiques sur des questions fédérales-provinciales, à savoir: demande au fédéral de réduire le taux d'intérêt, opposition au contrôle du fédéral sur les emprunts provinciaux et à son intrusion dans la voirie provinciale. En quelques mois seulement, se sont établies entre les premiers ministres des deux grandes provinces des relations aussi cordiales que fructueuses qui augmentent considérablement la force du Québec face à Ottawa en cette période troublée qui a bouleversé l'équilibre instable sur lequel repose le fédéralisme canadien.

La crise économique mondiale de 1929 à 1939 qui touche durement le Canada, montre la fragilité de l'économie canadienne et l'insuffisance des moyens dont disposent les provinces pour remplir leurs obligations. La crise fait prendre conscience à une élite anglo-canadienne et à certaines provinces de la nécessité, réelle ou imaginaire, d'un pouvoir central fort pour sortir le Canada du marasme économique et pour assurer à tous les citoyens canadiens un minimum de justice sociale. Ce mouvement d'opinion - ayant des objectifs autant économiques que politiques - la misère généralisée et la quasi-faillite de quelques provinces obligèrent le gouvernement fédéral (au temps de Bennett comme au temps de King) à adopter une politique centralisatrice. La bataille entre centralisateurs et autonomistes atteindra son paroxysme avec la création de la Commission Rowell-Sirois chargée d'enquêter sur les relations entre le Dominion et les provinces. Pour résister à ce très fort courant centralisateur qui déferle sur le pays, le Québec et l'Ontario établiront un front commun d'une ampleur exceptionnelle.

La coopération et les prises de position communes du Québec et de l'Ontario s'inscrivaient dans le cadre de la vie politique

31 M. Duplessis à M. F. Hepburn, 1er septembre 1936. Hepburn Papers, Private, 1936. P.A.O. 
normale de la fédération canadienne et n'avaient suscité aucun commentaire défavorable. Mais à partir d'avril 1937 et des mois qui suivront, la politique ouvrière et les critiques formulées contre le gouvernement fédéral par Hepburn et Duplessis provoquent une vive inquiétude en certains milieux.

Hepburn, lors d'une grève aux usines de General Motors à Oshawa, puis Duplessis, à l'occasion d'une grève dans l'industrie de la robe à Montréal, déclenchent une offensive contre de prétendus communistes et dénoncent avec virulence le gouvernement fédéral qu'ils accusent de ne pas prendre ses responsabilités contre les "agitateurs étrangers".

Duplessis, qui à cette époque vient de faire voter sa célèbre "loi du cadenas" - qu'Ottawa n'osera pas désavouer pour des raisons bien plus politiques que constitutionnelles - déclare:

Tant que je serai premier ministre de la province, je n'endurerai pas d'entrée officielle, officieuse, camouflée ou déguisée, de communistes dans Québec. C'est l'intention du gouvernement de maintenir l'ordre à tout prix dans la province, bien que l'abolition de l'article 98 du code pénal rende cette tâche difficile à accomplir. ${ }^{32}$

L'article 98 était une loi fédérale extrêmement sévère contre toute personne membre d'une organisation dite subversive, qui avait été votée par un gouvernement pris de panique lors de la grève générale de Winnipeg en $1919 .{ }^{33}$ Les libéraux dès leur retour au pouvoir en 1935 avaient abrogé cet article. Duplessis revient souvent sur la question communiste et il blâme toujours le fédéral.

Ce n'est pas nous qui avons le contrôle de la déportation. Je ne fais que de la politique provinciale. Je le dis en passant. Et ceux qui colportent que je vais à Ottawa se trompent. Mais nous n'avons pas la coopération nécessaire du gouvernement fédéral en matière ouvrière et de communisme. Et puis, il est temps que je le dise, il faut autre chose qu'un ministre de la justice [Ernest Lapointe] qui veut combattre les communistes avec les idées quand les communistes usent de pistolets et de canons. Nous allons faire pression sur Ottawa encore une fois. J'espère qu'on montrera le courage et l'énergie de $M$. Hepburn. ${ }^{34}$

32 Le Canada, 1er mai 1937.

33 A.R.M. Lower, Colony to Nation (Toronto, Third ed., 1957), 449.

${ }^{34}$ Le Devoir, 4 mai 1937. 
Les libéraux d'Ottawa comme de Québec s'abstiennent de commenter la politique de Hepburn et son alliance avec Duplessis, pour ne pas élargir le fossé qui sépare l'aile fédérale et l'aile ontarienne du parti libéral. Cependant, la chasse au communisme engagée par Duplessis, ses professions de foi autonomistes qui se transforment souvent en attaques contre les libéraux fédéraux commencent à agacer l'opposition québécoise comme on peut le constater lors d'un débat à l'Assemblée législative le 14 mai. Duplessis, ce jour-là, dresse un bilan des relations QuébecOttawa. Au début de son administration, dit-il, le gouvernement fédéral a collaboré avec celui du Québec. Mais assez rapidement, les bonnes relations se détériorèrent parce qu'Ottawa fit intervenir des considérations partisanes. Il est évident, selon le premier ministre, que la conférence fédérale-provinciale de décembre 1936 constituait une attaque centralisatrice d'Ottawa car celui-ci se proposait de contrôler les emprunts des provinces. Le Québec et l'Ontario unirent leurs forces pour repousser cette attaque insidieuse. Par la suite, le gouvernement fédéral a fait preuve de mauvaise volonté, notamment dans les négociations relatives aux pêcheries maritimes. Selon Duplessis, le Québec paie trente pour cent des impôts du Canada, et il est injuste qu'il ne reçoive aucune subvention pour ses pêcheries maritimes. Il faut dire que seul le Québec a pris à sa charge ce domaine; et ce, après une lutte de plusieurs années. Selon Duplessis, Ottawa a donné autorité à la province en ce domaine, simplement pour se débarrasser d'une charge financière. La situation est telle, qu'il a offert au fédéral de reprendre les pêcheries et après plusieurs mois, le gouvernement King ne s'est pas encore prononcé. L'opposition, qui n'est pas contre ce transfert de pouvoir, aura tout de même beau jeu de faire remarquer qu'il s'agit d'un curieux grief pour celui qui se veut grand défenseur de l'autonomie provinciale! Duplessis reproche aussi à Ottawa de ne pas prendre ses responsabilités quant au chômage et de ne pas donner au Québec sa juste part des subventions payées par le fédéral. Pour comble, Ottawa, par le moyen de ses maigres subventions, voudrait contrôler les finances provinciales. T.-D. Bouchard, le chef parlementaire de l'opposition, soutient que le fédéral veut ce pouvoir 
uniquement pour surveiller le gouvernement créditiste d'Alberta. Duplessis rétorque que si le principe du contrôle fédéral est accepté, il pourra s'appliquer à toutes les provinces sans exception. Bouchard ne pouvant réfuter cet argument, finit par dire qu'Ottawa a raison. Il va même jusqu'à comparer le contrôle du fédéral sur les provinces, au contrôle de celles-ci sur les municipalités! Duplessis, contrairement aux libéraux, préfère ne pas recevoir de subventions plutôt que de céder sur ce point. Accepter ce principe pourrait éventuellement nous conduire à l'union législative, et cela signifie que "Québec serait en minorité. Or nous n'avons pas besoin d'aller loin en dehors de notre province pour voir comment on traite les minorités $\gg .^{35}$ Finalement, le premier ministre reproche à Ottawa de ne pas collaborer avec la province dans la lutte contre le communisme. Duplessis termine son discours sur cette note menaçante: "Si Ottawa veut collaborer, j'en suis. Mais s'ils veulent (sic) la guerre, ils vont (sic) l'avoir".36

Peu après, dans un retentissant discours, Hepburn annonce sa rupture avec King et fait l'éloge de Duplessis. Mais les deux chefs libéraux, à l'approche des élections provinciales ontariennes, se réconcilieront. Hepburn remporte une impressionnante victoire en octobre 1937 et Duplessis s'en réjouit. Il réitère son désir de coopérer avec l'Ontario "pour le bien des deux provinces et du pays tout entier" ${ }^{37}$. La réconciliation King-Hepburn et les bonnes intentions exprimées par Duplessis ont dû plaire au président de la nouvelle Commission royale d'enquête sur les relations entre le Dominion et les provinces qui vient conférer avec le premier ministre du Québec. Après l'entrevue, le président Rowell assura les journalistes que le chef du gouvernement avait promis son entière collaboration à la commission. ${ }^{38}$

Le gouvernement King ne veut pas se contenter d'étudier les problèmes, il entend profiter de la conjoncture politique favorable en Ontario pour proposer un projet d'assurancechômage. Hepburn accepte l'idée d'amender la constitution pour

35 Ibid., 15 mai 1937.

36 Ibid., 15 mai 1937.

37 Ibid., 12 octobre 1937.

38 Ibid., 22 octobre 1937. 
donner à Ottawa le pouvoir d'intervenir dans ce domaine. Duplessis refuse. Il suggère qu'au lieu d'amender la constitution pour confier à Ottawa un pouvoir exclusif en cette matière, on organise un système basé sur des législations concurrentes dans chaque province.$^{39} \mathrm{King}$ rejette cette proposition parce que trop incertaine et instable. ${ }^{40}$ Duplessis estimant que ce domaine est et doit rester sous la juridiction provinciale non seulement s'opposera au projet mais encouragera Hepburn à l'imiter. ${ }^{41}$ Par la suite, le premier ministre d'Ontario reviendra sur sa première décision favorable au sujet d'amendement et l'opposition conjuguée du Québec et de l'Ontario paralysera l'action du gouvernement fédéral qui préférera abandonner le projet plutôt que de partager la responsabilité de l'assurance chômage avec les provinces.

Après les élections ontariennes d'octobre 1937, Hepburn, déçu par l'attitude d'Ottawa à son égard et inquiet par les témoignages présentés devant la Commission Rowell, décide de relancer l'alliance Québec-Ontario. Il déclare: "Nous allons contracter une alliance économique avec le Québec. Les exigences des provinces de l'Ouest semblent indiquer qu'il faudra que l'Ontario et le Québec s'entendent en ce qui concerne l'exposition de leurs vues à la Commission [Rowell]" 42 .

Du 13 décembre 1937 au 20 avril 1938, Duplessis et Hepburn se rencontrent à quatre reprises, soit à Montréal soit à Toronto, pour préparer leur stratégie face à la Commission d'enquête sur les relations entre le Dominion et les provinces.

Début mai, à dix jours d'intervalle, Hepburn au nom de l'Ontario, puis Me E. Beaulieu au nom du Québec, ${ }^{43}$ présentent leur point de vue à la Commission Rowell. La comparaison des mémoires des deux provinces centrales nous révèle à quel point les rencontres Duplessis-Hepburn, en vue de constituer

39 M. Duplessis à W. L. M. King, 23 novembre 1937. Cité dans R. Rumilly, Histoire de la province de Québec, 36: 219.

${ }_{40} \mathrm{~W}$. L. M. King à M. Duplessis, 26 novembre 1937. Cité dans R. Rumilly, Histoire de la province de Québec, 36: 220.

41 M. Duplessis à M. F. Hepburn, 31 janvier 1938. Hepburn Papers, Supplementary, Private, 1938. P.A.O.

42 Le Devoir, 11 décembre 1937.

43 Duplessis s'est contenté de déléguer un de ses conseillers juridiques. 
un front commun, ont été fructueuses. Quoi qu'en disent les deux premiers ministres, leur alliance dépasse de beaucoup la question économique, elle a un caractère éminemment politique. Il ne fait aucun doute que les forces conjuguées du Québec et de l'Ontario constituent un facteur d'une extrême importance dans l'évolution du fédéralisme canadien.

Les deux mémoires ${ }^{44}$ font savoir clairement qu'ils ne reconnaissent pas l'autorité de la Commission parce qu'elle est contraire à l'esprit de la Confédération, parce qu'elle a été instituée d'une manière non démocratique et, finalement, parce qu'elle est un instrument inapproprié pour résoudre les problèmes qui se posent entre le Dominion et les provinces.

Le Québec insiste davantage sur le premier point, car, selon lui, l'Acte de 1867 a le caractère d'un pacte entre États souverains. Par conséquent, chaque gouvernement étant souverain dans la sphère de ses attributions, le gouvernement central ne peut décider de sa seule autorité d'enquêter sur les administrations provinciales, pas plus qu'il ne peut amender ce pacte sans l'accord unanime de toutes les parties contractantes. Le mémoire québécois cite un passage du mémoire ontarien pour étayer son argumentation.

Le Québec va peut-être plus loin que l'Ontario lorsqu'il affirme que les pouvoirs résiduaires appartiennent aux provinces et qu'on ne peut modifier AUCUNE clause de la Constitution sans l'accord unanime des provinces.

Les deux mémoires consacrent plusieurs pages à dénoncer la centralisation comme étant un "mal national et social". MM. Duplessis et Hepburn qu'on a accusés de fascisme, prennent figure de défenseurs de la démocratie. Les deux soulignent que la centralisation se rencontre surtout dans les pays totalitaires. Non seulement la centralisation est-elle une menace pour la démocratie, mais, de plus, elle est inefficace dans un pays aussi vaste et aussi divers que le Canada.

Les provinces ont besoin de revenus suffisants pour exercer toutes leurs responsabilités et elles devraient avoir priorité dans

44 Report of the Royal Commission or Dominion-Provincial Relations. Report of Proceedings (Ottawa, 1938) 45: 7396-7452A et 51: 8129-8138. 
le champ de l'impôt direct. Et selon le mémoire ontarien, la démocratie exige que chaque gouvernement recueille et réponde de l'argent qu'il dépense; ce qui, d'ailleurs, amènera chaque province à vivre selon ses moyens.

Dans la deuxième partie de son mémoire, le premier ministre Hepburn s'en prend d'une manière directe à l'Ouest qui se plaint constamment d'être exploité par l'Est. Dans un plaidoyer passionné, il montre que c'est peut-être l'Est qui devrait récriminer. L'Ontario et le Québec, dit-il, n'ont pas à payer de leur argent et à sacrifier leur autonomie pour la politique absurde de l'Ouest et pour satisfaire des désirs qui ne correspondent pas à ses moyens.

Le Québec, sans développer ces idées, exprime clairement dans son mémoire qu'il partage pleinement les vues de l'Ontario relativement à cette question.

On demande la centralisation, dit Hepburn, afin d'assurer à tous les Canadiens des services sociaux uniformes. Cela est impossible et chaque province doit se résigner à vivre selon ses moyens. De plus, si on veut régler les problèmes sociaux du pays, il faut d'abord et avant tout développer l'économie canadienne.

Nous devons, poursuit le mémoire ontarien, assurer à chaque citoyen une vie décente tout en conservant à chaque province son autonomie de même que son héritage politique et culturel. Les provinces peuvent régler leurs problèmes sans recourir au fédéral, et la preuve en est la bonne entente et la coopération établies entre les provinces de Québec et d'Ontario qui, toutes deux, sont prêtes à collaborer aussi bien avec le gouvernement central qu'avec les autres gouvernements provinciaux.

À noter, en terminant, que l'Ontario a présenté un volumineux mémoire, tandis que Québec s'est contenté d'un texte d'une dizaine de pages qui s'appuie presque exclusivement sur des arguments juridiques. Même si Duplessis ne reconnaissait pas l'autorité de la Commission et même s'il partageait les idées émises par son ami Hepburn qu'il cite à quatre reprises dans son mémoire, il aurait peut-être pu imiter le premier ministre 
W. Aberhart d'Alberta, c'est-à-dire préparer un mémoire bien documenté sur le cas du Québec, et le présenter à la population.

Les deux premiers ministres plaident en faveur d'une fédération décentralisée en s'appuyant malheureusement sur une conception de la Constitution dont plusieurs à l'époque contestent - non sans raison - la validité. Plus grave encore, la philosophie sociale et économique qui se dégage de ces mémoires des deux provinces "riches" évoque plus le libéralisme du XIXe siècle que le "Welfare State" que demande une grande partie de la population canadienne.

À la suite de cette réaction non équivoque de l'alliance Duplessis-Hepburn, Léopold Richer, dans Le Devoir, signale qu'Ottawa est inquiet. D'autant plus, comme il le souligne, qu'aucun gouvernement ne peut se passer en même temps, de l'appui des deux provinces centrales. ${ }^{45}$

Quoi qu'on puisse penser de la valeur de l'autonomie provinciale, on reconnaîtra que Duplessis a su mettre en œuvre une stratégie extrêmement efficace pour contrer l'évolution du fédéralisme canadien vers une plus grande centralisation.

La guerre sera la pierre d'achoppement de l'alliance Duplessis-Hepburn. Le premier ministre d'Ontario fait preuve d'un zèle impérialiste très poussé et Duplessis, premier ministre du Québec, ne peut le suivre sur ce terrain. Pendant que l'un blâme Ottawa de ne pas faire assez pour la défense, l'autre l'accuse de faire trop.

Après l'entrée en guerre du Canada, Duplessis décide de retourner devant l'électorat, même si son mandat n'est pas terminé, car dit-il:

Invoquant le prétexte de la guerre, déclarée par le gouvernement fédéral, une campagne d'assimilation et de centralisation, manifeste depuis plusieurs années; s'accentue de façon intolérable.

Des arrêtés ministériels ont été passés par Ottawa en vertu du "War Measures Act" ou Mesures de guerre", avec le désir et l'effet de centraliser à Ottawa pour des fins de

${ }^{45}$ Le Devoir, 13 mai 1938. 


\begin{abstract}
guerre, toute la finance des particuliers, des municipalités, des provinces et du pays.

Le gouvernement de l'Union nationale a formé une administration composée de libéraux, de conservateurs et d'indépendants (...) qui a à cœur [de défendre] ... l'autonomie provinciale (...) parce qu'elle constitue, (...) le rempart le plus solide de nos institutions, de nos traditions les plus chères et de nos droits fondamentaux. ${ }^{46}$
\end{abstract}

Les libéraux provinciaux accusent le chef de l'Union Nationale de profiter de la guerre pour faire appel aux préjugés de la population et tenter par la même occasion de camoufler l'état délabré des finances provinciales. Pour eux, le fait de ne pouvoir utiliser le crédit de la province ni même l'obligation pour le premier ministre du Québec de soumettre le texte de ses discours à un censeur fédéral avant de pouvoir s'adresser à l'électorat par le truchement de la radio, ne constituent pas des menaces à l'autonomie provinciale! Bref, ils entendent faire l'élection sur les vrais problèmes de l'administration provinciale, et non sur les questions de compétence fédérale. À Ottawa, les ministres fédéraux du Québec (Lapointe, Cardin et Power) écartent d'un revers de la main cette stratégie douteuse. Selon eux, l'objet de l'élection, ce n'est pas l'autonomie provinciale, mais la conscription. Les fédéraux, qui se disent "le rempart contre la conscription", avertissent la population que si Duplessis est reporté au pouvoir, ils interpréteront ce geste comme un vote de non-confiance à leur égard. Par conséquent, ils démissionneront du cabinet fédéral et automatiquement, ce sera la conscription. C'est ainsi qu'un vote pour Duplessis, aussi incroyable que cela puisse paraître, devient un vote pour la conscription ! Ce chantage, d'une extraordinaire efficacité, dans le contexte de l'époque, plus le "patriotic money" de Toronto et d'ailleurs, ${ }^{47}$ scelleront le sort de Duplessis.

Duplessis, pendant cette campagne électorale, sans rien ajouter à sa conception de l'autonomie provinciale, sait trouver des mots simples et un accent nouveau pour en parler:

J'entends que l'on sache à Ottawa, que nous sommes maîtres

chez nous, que nous voulons rester maîtres chez nous. ${ }^{48}$

46 Ibid., 25 septembre 1939.

47 N. Ward, ed., The Memoirs of Chubby Power. A Party Politician (Toronto, 1956), 125-128.

48 Le Devoir, 5 octobre 1939. 
L'autonomie c'est le droit pour la province de s'administrer. Violer l'autonomie de la province ce serait comme si la ville de Rivière du Loup était administrée par Winnipeg ou Vancouver. ${ }^{49}$

Nous vous demandons peuple de Québec de vous tenir debout contre les tyrans d'Ottawa (...) de vous dresser devant eux pour que la province de Québec puisse vivre et respirer l'air de la liberté...50

Les libéraux, qui depuis vingt ans triomphent des conservateurs en brandissant le spectre de la conscription, réussissent une fois de plus à mystifier la population québécoise.

Duplessis est vaincu mais pas nécessairement la cause qu'il a tenté de défendre. La preuve en est qu'il sera premier ministre du Québec de 1944 à 1959 !

RENÉ DUROCHER

Université de Montréal

49 Ibid., 9 octobre 1939.

50 Ibid., 13 octobre 1939.

\section{LIVRES REÇUS :}

Le Canada au seuil du siècle de l'abondance - entretiens de Cerisy-la-Salle 1968. Editions HMH Ltée, 380 ouest, rue Craig, Montréal 126. 376 pages. $\$ 8.00$.

Comprend 29 collaborateurs. Séance après séance, texte intégral des communications, résumé des discussions qui ont suivi, réduites à l'essentiel. Inventaire, suivant les méthodes les plus rigoureuses, des questions auxquelles les Canadiens auront à faire face dans les prochaines années.

Littérature canadienne-française - "Conférences J.-A. de Sève", 1-10. 346 p. Les Presses de l'Université de Montréal, c.p. 6128, Montréal 101. Textes de Jacques Godbout, Gérard Tougas, David M. Hayne, Glen Shortliffe, Gilles Marcotte, Jean Ethier-Blais, Jacques Brault, Albert Le Grand, Michel van Schendel, Jean-Charles Falardeau et G.-André Vachon, suivis d'une Bibliographie de la critique québécoise (19641968). $\$ 4.50$. 